\title{
The Morphologic Profile of Inflammatory Bowel Disease and the Diagnostic Problem of Crohn's Disease versus TB Colitis - A Case Series
}

\author{
Maria Lourdes Tilbe, Francia Victoria De Los Reyes, Ricka Cu, Kathleen Perez
}

University of the East Ramon Magsaysay Memorial Medical Center, Quezon City, Philippines

\section{ABSTRACT}

The aim of this study is to describe the morphologic profile of the biopsy and resection specimen that were diagnosed with Crohn's disease and ulcerative colitis in the University of the East Ramon Magsaysay Memorial Medical Center (UERMMMC) from 2008-2016. Features that classify the specimen as Inflammatory Bowel Disease - Indeterminate Type are also presented. Considerations for the definitive IBD classification after an initial indeterminate diagnosis by morphology are also briefly discussed. Biopsy and resection specimen that were diagnosed with Crohn's disease, cannot exclude TB Colitis, are also presented; and the subsequent steps for a definitive classification are also discussed. All the patients included underwent an endoscopic biopsy, and are categorized by histopathologic diagnoses, age, sex, and GIT segment involved in the endoscopic procedure. Patients that underwent subsequent resection due to the disease condition are also identified.

Comparison of the histologic findings observed in the patients, with the microscopic basis for the diagnosis recommended by the European consensus on the histopathology of inflammatory bowel disease (2013), and with the histologic features described by Patil et al., (2015) for the inflammatory disorders of the large intestine, is done. The histologic features described by Lamps (2015) for the gastrointestinal TB is used in the evaluation of the findings in the patients diagnosed with Crohn's disease, cannot exclude TB Colitis.

There are 5 Crohn's disease patients, accounting for $0.8 \%$ of all patients with lower GIT inflammatory conditions, and 10 ulcerative colitis patients, accounting for $1.6 \%$ of all patients with lower GIT inflammatory conditions. Seven patients, which comprise $1.1 \%$ of all patients with lower GIT inflammatory conditions, have the diagnosis of indeterminate colitis. The histologic features of 6 out of 7 patients that had the initial diagnosis of indeterminate colitis presented with morphologic features that favored an ulcerative colitis, but with Crohn's disease features. In comparison, one patient who had an initial diagnosis of indeterminate colitis presented with morphologic features that favored Crohn's disease but with ulcerative colitis features. In these patients, correlation with chronology of symptoms and associated ancillary procedures that can classify the patients as CD or UC are recommended to the gastroenterologist attending such patients so that a more definitive classification can be done.

Four patients, accounting for $0.6 \%$ of all lower GIT inflammatory conditions, were initially diagnosed as Crohn's disease, cannot exclude TB Colitis. This is in contrast with 34 patients who were diagnosed with Chronic Granulomatous Inflammation, Tuberculosis which accounted for $5.7 \%$ of all patients that were diagnosed with inflammatory conditions of the lower GIT. The remaining 536 patients were composed of acute self-limited/ infectious colitis, ischemic colitis, eosinophilic colitis, inflammatory polyp, and nonspecific inflammation.

With the trend of increasing incidence of Inflammatory Bowel Disease in Asia, comparison of the more commonly seen causes of chronic inflammation of the gastrointestinal tract with a condition that appears to have a growing incidence in the region is necessary for optimal diagnostic protocol, management, and quality of care.

Key words: inflammatory bowel disease, Crohn's disease, ulcerative colitis, indeterminate colitis, intestinal tuberculosis, colitis, morphology, histopathology

\section{ISSN 2507-8364 (Online)}

Printed in the Philippines.

Copyright(C) 2017 by the PJP.

Received: 1 April 2017.

Accepted: 3 June 2017

Published online first: 7 August 2017.

https://doi.org/10.21141/PJP.2017.013

Corresponding author: Francia Victoria A. De Los Reyes, MD

E-mail: kaidelosreyes@gmail.com

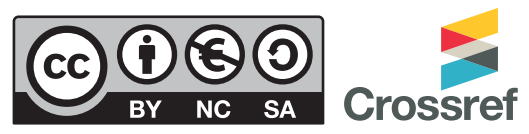

\section{INTRODUCTION}

Inflammatory bowel disease (IBD), comprising of the two disorders ulcerative colitis (UC) and Crohn's disease (CD), is a disease condition that results from a chronic, inappropriate immune activation in the mucosa with subsequent involvement of the submucosa for ulcerative colitis, and full-thickness wall involvement for Crohn's disease. Although known to be more frequent in North America, northern Europe, and Australia, there is a trend of increasing incidence documented in Asia, Africa, and South America.

Hypothesis regarding the role of the luminal gut microbiota and the changes in gut microbiome composition due to improved 
food storage condition, and decreased food contamination of previously documented commonly occurring food-borne infectious organisms have been described. The role of changes in the gut microbial flora triggers a cascade of persistent inflammation in susceptible hosts that manifest as chronic gastrointestinal symptoms. Although, much is yet to be discovered regarding the mechanisms of this. ${ }^{1}$

Intestinal tuberculosis (TB), on the other hand, is a disease condition that has a high disease burden in developing countries. To apply this, in the Philippines, TB is the sixth leading cause of morbidity and mortality, and is the ninth out of the 22 highest TB-burden countries in the world. The country likewise has one of the highest burdens of multidrug-resistant TB. ${ }^{2}$ Intestinal TB belongs under the broader category of abdominal TB, which has been documented to constitute about $12 \%$ of extra-pulmonary $\mathrm{TB}$ and $1 \%-3 \%$ of the total TB cases. ${ }^{3}$ However, no multicenter study in the country documenting the trend of incidence has been documented to specify the incidence or prevalence of intestinal $\mathrm{TB}$ alone versus all other abdominal TB.

Both of the two disease conditions, IBD and intestinal TB have features constituting chronic inflammation, segmental or diffuse involvement, prolonged treatment requirement, and significant interplay of host immune-response with gut microbiomerelated injury. Comparatively, it has been documented that there is an overlap in the genes responsible for the immunemediated mechanism of IBD and the immune response to mycobacteria infection, including Mycobacterium tuberculosis and Mycobacterium leprae. ${ }^{1,4}$ As such, comparison of the morphologic features of IBD and intestinal TB may be necessary in countries where there is an increasing incidence of one and a consistently high incidence of the other.

To address such concern in our local setting, this paper describes the morphologic features of the biopsy and/or resection specimen of the patients diagnosed with $\mathrm{CD}, \mathrm{UC}$, and IBD-Indeterminate (IBD-IC), and CD vs TB colitis in the University of the East Ramon Magsaysay Memorial Medical Center from 2008-2016. The aim of comparing the morphologic features of IBD and indicating instances when TB cannot be completely excluded is so that there is an initial groundwork for establishing an algorithm that can be used in diagnosing IBD and excluding $\mathrm{TB}$, or concluding a concurrent $\mathrm{TB}$ infection given a particular morphologic pattern.

\section{CASE}

To determine the prevalence of IBD in our institution, we reviewed all the surgical pathology reports in the UERMMMC Pathology Laboratory database from 2008-2016 and determined the occurrence of the various morphologic diagnosis of the inflammatory conditions of the lower gastrointestinal tract. The census was generated using the morphologic diagnosis that was reported in our institution for the past 8 years, and were compared with the morphologic findings used by Odze et al., in

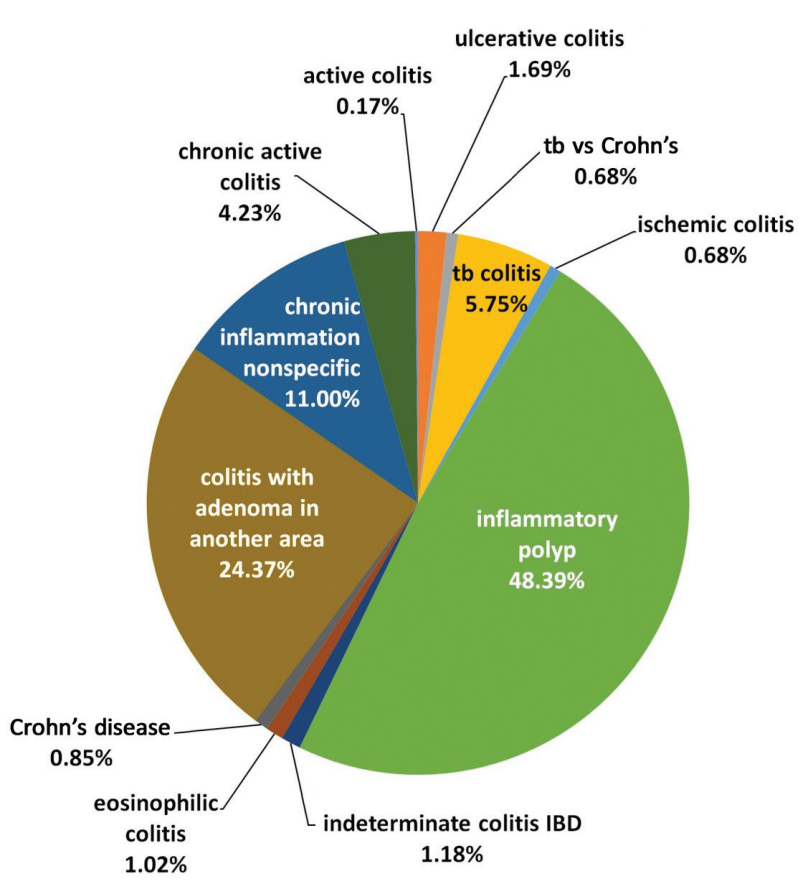

Figure 1. Inflammatory diseases of the ileum, colon, and anorectal region in the UERMMMC Pathology Laboratory from 2008 to $2016(n=596)$.

2015 discussing the disorders of the GIT to ensure the timeliness of reporting such morphologic features. The census included inflammatory polyp, chronic nonspecific inflammation, active colitis, chronic active colitis, ischemic colitis, eosinophilic colitis, diagnosis of colitis with an adenoma seen in another segment of the GIT but with no malignancy, TB colitis, CD, UC, IBDindeterminate, and $\mathrm{CD}$ but $\mathrm{TB}$ colitis cannot be completely excluded. All reports with the surgical pathology diagnosis of acute appendicitis, ruptured viscus that was not secondary to a known chronic inflammatory condition, post-operative complications, diverticular disease, and malignancy-associated lesions without a previously documented association with IBD, were excluded. The patients with the diagnosis of CD, UC, IBDindeterminate, $\mathrm{CD}$ but cannot exclude $\mathrm{TB}$ were described in terms of the male-to-female ratio, age, and GIT segment involved in the endoscopic or surgical resection procedures (Table 1).

Comparison of the morphologic findings of the included reports, with the microscopic basis for the diagnosis recommended by the European consensus on the histopathology of inflammatory bowel disease of $2013,{ }^{5}$ and with the histologic features described by Patil et al., in 2015 for the inflammatory disorders of the large intestine, and the histologic features described by Lamps in 2015 for the gastrointestinal TB, were done.

Using these parameters, a total of 596 patients that were diagnosed with inflammatory conditions of the lower gastrointestinal tract were included in the census.

Table 1. Characteristics of IBD patients and intestinal TB vs. CD patients

\begin{tabular}{|c|c|c|c|c|}
\hline Diagnosis & Number of Cases & Age Mean (Range) & $\mathrm{M} / \mathrm{F}$ & Site of Involvement by Frequency \\
\hline Crohn's Disease & 5 & $35.4(17-74)$ & $5 / 0$ & ileum > stomach, cecum, ascending colon, descending colon, rectum \\
\hline Ulcerative Colitis & 10 & $49(25-77)$ & $5 / 5$ & $\begin{array}{l}\text { sigmoid colon }>\text { rectum }>\text { descending colon }>\text { ascending colon, } \\
\text { transverse colon }>\text { cecum, anastomotic site }(s / p \text { right hemicolectomy })\end{array}$ \\
\hline Indeterminate Colitis & 7 & $48.8(17-72)$ & $5 / 2$ & cecum $>$ rectum $>$ ileum, transverse colon, sigmoid colon $>$ anal canal \\
\hline $\begin{array}{l}\text { Intestinal TB vs. } \\
\text { Crohn's Disease }\end{array}$ & 4 & $39.5(26-66)$ & $4 / 0$ & $\begin{array}{l}\text { lleum > cecum, ascending colon, transverse colon, descending colon, } \\
\text { rectum }\end{array}$ \\
\hline
\end{tabular}




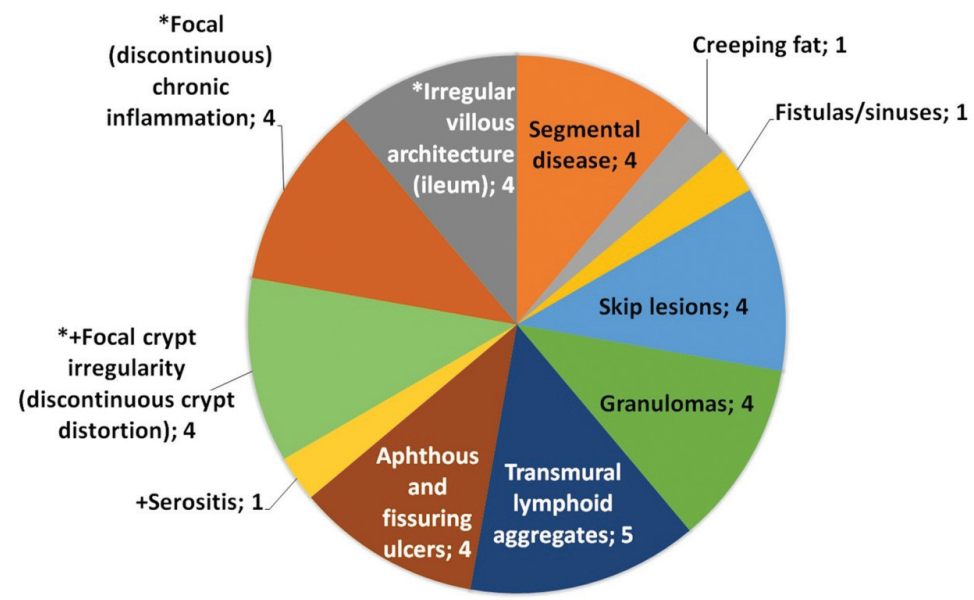

Figure 2. Frequency of specific morphologic features in Crohn's disease $(n=5)$.

+ minor morphologic criteria Patil et al., 2015.

* morphologic criteria European Consensus on the histopathology of Crohn's disease, 2013

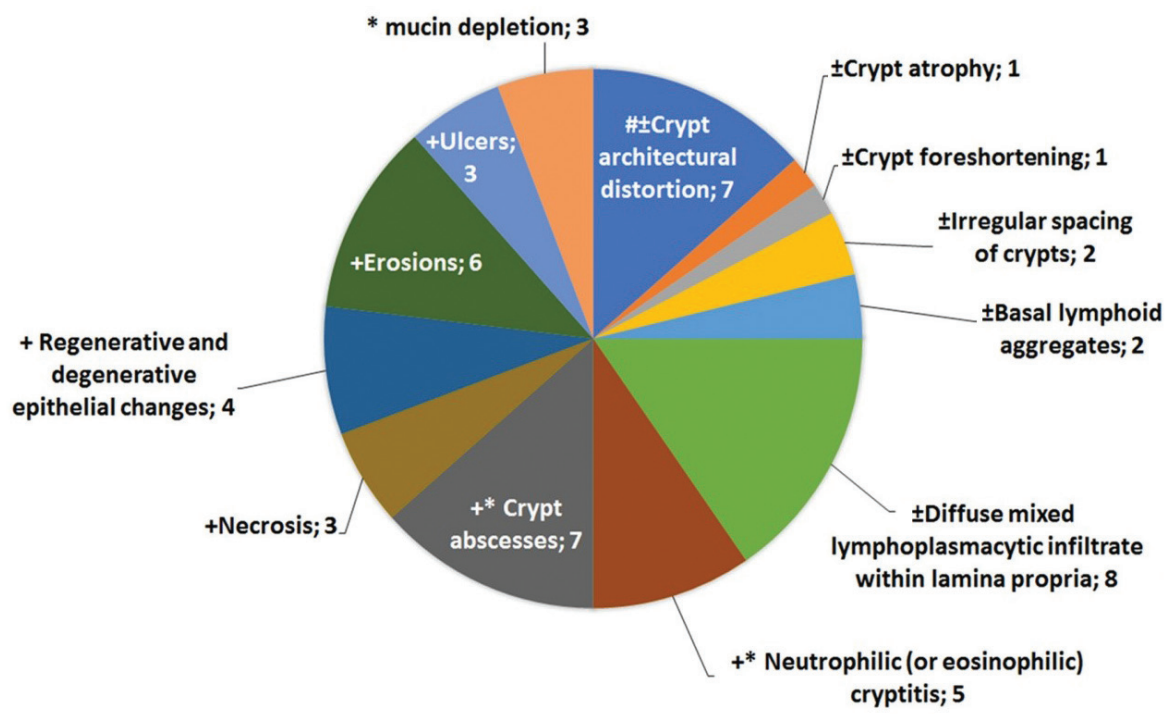

Figure 3. Frequency of specific morphologic features in ulcerative colitis $(n=10)$.

\pm histologic features of chronicity of UC lesion Patil et al., 2015,

+ histologic features of activity of UC Patil et al., 2015,

* morphologic criteria for active European Consensus on the histopathology of ulcerative colitis, 2013

Among all of these, only 5 patients were diagnosed with Crohn's disease, accounting for $0.8 \%$ of all included patients. The patients diagnosed with Crohn's disease were all male, with the age range of 17-74 years old, and the sites involves are the following in descending order of frequency: ileum in 4 patients, and stomach, cecum, ascending colon, descending colon, and rectum with involvement found in 1 patient each. The most common major pathologic findings include transmural lymphoid aggregates, segmental disease, granulomas, fistulas, and fissuring ulcers. Only focal crypt irregularity among the secondary pathologic features is consistently observed. All the major pathologic features indicated by Patil et al., and all the features enumerated under the 2013 consensus are observed in the patients (Figure 2).

In contrast to $\mathrm{CD}$, ulcerative colitis was observed in 10 patients, and this comprised $1.6 \%$ of the population included. In this group, there is a 1:1 male-to-female ratio, and the age range is similarly wide like in $\mathrm{CD}$, with the patients' ages ranging from 25 to 77 years old. The sites involved are the following, in descending order of frequency: sigmoid colon with 6 patients noting involvement, rectal involvement in 5 patients, descending colon involvement in 3 patients, ascending colon and transverse colon involvement in 2 patients, cecal involvement in 1 patient, and an anastomotic site in a status post right hemicolectomy patient in 1 patient. For all patients diagnosed with ulcerative colitis, the most commonly observed features include diffuse mixed lymphocytic and plasmacytic infiltrate within the lamina propria, crypt abscess, and crypt architectural distortion. All of the patients presented with a chronic active type of disease with no fibrosis observed (Figure 3).

Seven patients, comprising $1.1 \%$ of the included population, were given the diagnosis of indeterminate colitis. Among the patients diagnosed with Inflammatory Bowel Disease-indeterminate colitis, the age range is 17-72 years old, and a 2.5:1 male-tofemale ratio was noted. The sites involved in descending order of frequency included cecum in 4 patients, rectum in 3 patients, ileum in 2 patients, transverse colon in 2 patients, sigmoid colon 
in 2 patients, and anal canal in 1 patient. The histologic features of 6 out of 7 indeterminate colitis favored ulcerative colitis, and only one patient had a morphologic diagnosis that favored Crohn's disease more than ulcerative colitis.

Although it is important to note that indeterminate colitis is an interim diagnosis that requires further work-up for a definitive classification, it is equally important for us to determine what kind of pitfalls were present during the histopathologic evaluation of such specimen and identify the morphologic features that precludes the definitive classification of GD or UC. Morphologic Diagnosis of IBD-Indeterminate type in the UERMMMC is an interim diagnosis and made only after exclusion of all other causes of colitis and the biopsy clearly has morphologic features pertaining to an IBD morphology, and when only one or two features of chronicity and one or two features of activity for ulcerative colitis were observed.

The most commonly observed features that caused the diagnosis of indeterminate colitis were divided into ulcerative colitis with Crohn's-like features, and Crohn's disease with ulcerative colitislike features. Crohn's disease with UG-like features were noted as persistently superficial fissuring ulcers in a discontinuous disease with anal fistula formation, but with no features indicating granuloma formation, transmural lymphoplasmacytic inflammation, or lymphoid hyperplasia. In contrast, UC with CD-like features were noted in those with superficial mucosal involvement which manifests as mucosal ulceration with absent granuloma formation but is discontinuous and has lymphoid hyperplasia with no cryptitis. In these patients, correlation with the clinical course and ancillary laboratory tests that can provide information whether the associated features of the patient's gastrointestinal lesion were more likely associated with $\mathrm{CD}$ or UC were done in coordination with the attending gastroenterologist so that a more definitive classification can be made. In patients with more morphologic features consistent with $\mathrm{CD}$, treatment for $\mathrm{CD}$ was initiated by the attending gastroenterologist, and treatment for $\mathrm{UG}$ was initiated in patients with more morphologic features consistent with UC. In indeterminate colitis, patients with CD-like lesions but lack granuloma or transmural inflammation may have undergone biopsy prior to the transmural involvement or development of granuloma of the segment sampled. In comparison, patients with UG-like features but have patchy disease may belong to the small population of UC with an initially segmental disease because there is limited involvement of particular colon segments, for example a diffuse involvement of the left side of the colon with a patchy involvement of the right side of the colon, which will become confluent towards the progression of $\mathrm{UC}$ into a diffuse involvement.

Also documented were 4 patients, accounting for $0.6 \%$ of all the patients included, have the diagnostic problem of CD, cannot totally rule out $\mathrm{TB}$ colitis. This is interpreted in the setting of a greater prevalence of clear cut TB colitis patients with 34 patients, comprising of $5.7 \%$ of all included patients, diagnosed with intestinal TB. The work-up of all patients with the morphologic diagnosis of $\mathrm{CD}$ cannot exclude TB were reviewed for the history of active or latent TB, status of ancillary testing for Mycobacterium TB such as PCR of endoscopic biopsy specimen and AFP special stain, taken at the same time as the specimen with the problematic diagnosis. In these patients, the age range is 2666 years old, all patients are males, and the sites involves are the following in descending order of frequency: ileum in 3 patients, and 1 patient each for cecum, ascending colon, transverse colon, descending colon, and rectum. All four patients with chronic granulomatous inflammation, consider TB colitis versus Crohn's disease show focal and discontinuous chronic inflammation. No discontinuous crypt distortion and no irregular villous architecture were seen. One patient also showed a lesion in the ileum with granulomas with multinucleated giant cells. In such situation, the consideration of intestinal TB was entertained due to the high incidence of the disease in the country. All the biopsy specimen also showed no Mycobacterium tuberculosis bacilli on AFB stain. Clinical evaluation also showed no evidence of an acute or latent TB infection in all four patients, and as such were managed for $\mathrm{CD}$.

However, one patient that had been undergoing treatment for CD for three months presented with an exacerbation of CD and underwent right hemicolectomy. The resection specimen showed positive Mycobacterium tuberculosis bacilli in the areas exhibiting caseation necrosis, whereas the biopsy showed negative findings on MTB PCR and AFB special stain in the specimen from the cecum and the rectum which was done three month prior to the exacerbation. In this patient, there was noncaseation necrosis in the biopsy specimen of the cecum, and in the subsequent sampling of the cecum after right hemicolectomy was done, but caseation necrosis was noted in the transverse colon segment of the resection specimen. The conversion from a previously negative to a positive TB involvement may indicate a latent TB infection that was activated upon immunosuppression during the course of treatment for Crohn's disease. ${ }^{6}$

\section{DISCUSSION}

The diagnosis of IBD requires the correlation of endoscopic findings that provides information on the gross features of the intestinal architecture, such as luminal caliber, distensibility, and macroscopic mucosal lesions, with the morphologic features noted in the histopathologic evaluation because even grossly unremarkable looking mucosa may harbor critical features that clinch the diagnosis of CD or UC. It is equally important to emphasize that multiple slide sections from the sampled segments are required and although there is no consensus as of the publication of Langer et al., in 2014 regarding the number of sections required for an adequate diagnosis, the recommendation of step-sections of two to three tissue levels, having five or more sections is useful to exhaust the features which may otherwise not be visualized if there is a limited sampling. ${ }^{7}$ This is particularly useful in our institution when faced with vague features such as poorly formed granulomas, or noncaseating granulomas in a resection specimen with another lesion that has a caseating granuloma.

Extraintestinal manifestations of IBD are also important but more so because having a particular IBD increases the chance of a patient developing a disease associated with one type versus another, for example Primary Sclerosing Cholangitis is more likely if the patient has ulcerative colitis than if the patient has Crohn's disease. Although if a patient presents with symptoms of rectal bleeding, mucoid discharge from the rectum, frequent stools, and associated liver disease manifesting as cholestasis, the diagnosis of ulcerative colitis is highly likely and quite warranted. However, the cornerstone of diagnosing IBD is still clinical constellation of symptoms, endoscopic findings, and morphologic features. As such, documenting the morphologic features that allows institutions to create an algorithm that can streamline the evaluation of chronic inflammatory lesions that 
may have mimics is necessary so that a standardized system based on morphologic parameters established in published literature can be used. Also, documenting problematic situations unique to regions with infectious processes that mimics IBD or complicates IBD may help in addressing future concerns related to such mimics. The standardized system based on morphologic parameters should incorporate ruling out TB when the classic morphology of TB is not seen.

Although this study is limited by the lack of information on the gross findings seen on endoscopic evaluation, the inclination of this study is to establish the morphologic features based on histopathology and to discuss the features that influence favoring the diagnosis of one specific lesion versus another when a case is under a larger general category.

Future studies to determine the incidence of $\mathrm{CD}$ and $\mathrm{UC}$, and its association with $\mathrm{TB}$ is recommended to determine whether there is an increasing trend of IBD and whether IBD treatment will lead to a higher occurrence of $\mathrm{TB}$ reactivation.

\section{CONCLUSION}

Given that there is an increasing trend of IBD in Asia, there should be a working system to classify such patients based on standardized morphologic parameters based on published literature that also reflect the current practices in areas with a higher incidence of IBD. Likewise, the algorithm for diagnosis should incorporate ruling out TB when the classic morphology of $\mathrm{TB}$ is not seen, and should address the development of active TB in those with latent TB infection that had undergone treatment for IBD. With the trend of increasing incidence of Inflammatory Bowel Disease in Asia, comparison of the more commonly seen causes of chronic inflammation of the gastrointestinal tract, particularly $\mathrm{TB}$, with a condition that appears to have a growing incidence in the region, such as IBD, is necessary for optimal management and improved quality of care.

\section{ACKNOWLEDGMENTS}

The authors would like to acknowledge the support of the faculty and staff of the Department of Pathology, College of Medicine, University of the East Ramon Magsaysay Memorial Medical Center, and the Section of Histopathology, Pathology Laboratory of the University of the East Ramon Magsaysay Memorial Medical Center.

\section{ETHICAL CONSIDERATION}

This case report has no studies performed to animal or human participants. This case report includes only the specimen submitted by the patient for surgical histopathology evaluation to the section of anatomic pathology with full, informed consent of specimen evaluation and non-profit academic discussion or report.

\section{STATEMENT OF AUTHORSHIP}

All authors certified fulfillment of ICMJE authorship criteria.

\section{AUTHOR DISCLOSURE}

The authors have declared no conflict of interest.

\section{FUNDING SOURCE}

None.

\section{REFERENCES}

1. Turner JR. The Gastrointestinal Tract. In: Kumar V, Abbas AK, Aster JC ed. Robbins and Cotran Pathologic Basis of Disease, 9th ed. Philadelphia: Elsevier-Saunders; 2015: 796-800.

2. Vianzon R, Garfin AMC, Lagos A, Belen R. The tuberculosis profile of the Philippines, 2003-2011: advancing DOTS and beyond. Western Pacific Surveillance and Response Journal. 2013;4(2):11-6. https://doi.org/10.5365/ wpsar.2012.3.4.022.

3. Sheer TA, Coyle WJ. Gastrointestinal tuberculosis. Curr Gastroenterol Rep. 2003;5(4):273-8. PMID: 12864956.

4. McNees A, Markesich D, Zayyani N, Graham D. Mycobacterium paratuberculosis as a cause of Crohn's disease. Expert Rev Gastroenterol Hepatol. 2015;9(12):1523 34. PMCID: PMC4894645. NIHMSID: NIHMS787513. https://doi.org/10.1586/17474124.2015.1093931.

5. Magro F, Langner C, Driessen A, et al. European consensus on the histopathology of inflammatory bowel disease. J Crohns Colitis. 2013;7(10):827-51. https://doi.org/10.1016/j. crohns.2013.06.001.

6. Ye L, Liu J, Lin Z, Gao Q. Does infliximab therapy increase incidence of tuberculosis in patients with inflammatory bowel disease in an endemic area: a nationwide study from China. Poster Presentation to the 12th Congress of European Crohn's and Colitis Organization, Barcelona 2017. https://www.ecco-ibd.eu/index.php/publications/ congress-abstract-s/abstracts-2017/item/p568-doesinfliximab-therapy-increase-incidence-of-tuberculosis-inpatients-with-inflammatory-bowel-disease-in-an-endemicarea-a-nationwide-study-from-china-2.html.

7. Langer C, Magro F, Driessen A, Ensari A, et al. The histopathological approach to inflammatory bowel disease: a practice guide. Virchows Arch. 2014;464(5):511-27. PMID: 24487791. https://doi.org/10.1007/s00428-014-1543-4.

Disclaimer: This journal is OPEN ACCESS, providing immediate access to its content on the principle that making research freely available to the public supports a greater global exchange of knowledge. As a requirement for submission to the PJP, all authors have accomplished an AUTHOR FORM, which declares that the ICMJE criteria for authorship have been met by each author listed, that the article represents original material, has not been published, accepted for publication in other journals, or concurrently submitted to other journals, and that all funding and conflicts of interest have been declared. Consent forms have been secured for the publication of information about patients or cases; otherwise, authors have declared that all means have been exhausted for securing consent. 